\title{
Linear operators on the space of bounded continuous functions with strict topologies
}

\author{
Marian Nowak
}

Received: 15 October 2009 / Accepted: 22 May 2010 / Published online: 23 June 2010

(C) The Author(s) 2010. This article is published with open access at Springerlink.com

\begin{abstract}
Let $X$ be a completely regular Hausdorff space, and let $C_{b}(X)$ denote the Banach space of all real-valued bounded continuous functions on $X$. We study linear operators from $C_{b}(X)$ provided with the strict topology $\beta_{\sigma}$ to a Banach space $\left(E,\|\cdot\|_{E}\right)$. In particular, we derive a Yosida-Hewitt type decomposition for weakly compact operators from $C_{b}(X)$ to $E$.
\end{abstract}

Keywords Space of bounded continuous functions - Strict topologies - Baire measures $\cdot \sigma$-Dini topologies $\cdot$ Weakly compact operators $\cdot$ Yosida-Hewitt decomposition · Generalized DF-space

Mathematics Subject Classification (2000) $\quad$ 46A70 - 46E10 - 46E27 · 47B38

\section{Introduction and terminology}

For terminology concerning vector lattices we refer to [1]. We denote by $\sigma(L, K)$ and $\tau(L, K)$ the weak topology and the Mackey topology on $L$ with respect to a dual pair $\langle L, K\rangle$. By $\mathbb{N}$ and $\mathbb{R}$ we denote the sets of natural and real numbers. From now on we assume that $(E,\|\cdot\|)$ is a real Banach space, and let $E^{\prime}$ and $E^{\prime \prime}$ stand for the Banach dual and the Banach bidual of $E$, respectively.

We assume that $X$ is a completely regular Hausdorff space. Let $C_{b}(X)$ be the Banach space of all real-valued bounded continuous functions on $X$ endowed with the supremum norm $\|\cdot\|_{\infty}$. Then the Banach dual $C_{b}(X)^{\prime}$ of $C_{b}(X)$ with the natural

M. Nowak $(\varangle)$

Faculty of Mathematics, Computer Science and Econometrics, University of Zielona Góra, ul. Szafrana 4A, 65-516 Zielona Gora, Poland e-mail: M.Nowak@wmie.uz.zgora.pl 
order $\left(\Phi_{1} \leq \Phi_{2}\right.$ if $\Phi_{1}(u) \leq \Phi_{2}(u)$ for each $\left.0 \leq u \in C_{b}(X)\right)$ is a Dedekind complete Banach lattice. By $C_{b}(X)^{\prime \prime}$ we will denote the Banach bidual of $C_{b}(X)$.

Let $\mathcal{B}$ (respectively, $\mathcal{B} a$ ) be the algebra (respectively, $\sigma$-algebra) of Baire sets in $X$, which is the algebra (respectively, $\sigma$-algebra) generated by the class of all zero-sets of functions of $C_{b}(X)$. Let $M(X)$ stand for the space of all Baire measures on $\mathcal{B}$. Then $M(X)$ with the norm $\|\mu\|=|\mu|(X)$ (= the total variation of $\mu)$ and the natural order $\left(\mu_{1} \leq \mu_{2}\right.$ if $\mu_{1}(A) \leq \mu_{2}(A)$ for all $\left.A \in \mathcal{B}\right)$ is a Dedekind complete Banach lattice. Due to the Alexandroff representation theorem (see [20], [21, Theorem 5.1]) $C_{b}(X)^{\prime}$ can be identified with $M(X)$ through the lattice isomorphism $M(X) \ni \mu \mapsto \Phi_{\mu} \in C_{b}(X)^{\prime}$, where $\Phi_{\mu}(u)=\int_{X} u(x) d \mu$ for all $u \in C_{b}(X)$, and $\left\|\Phi_{\mu}\right\|=\|\mu\|$.

A functional $\Phi \in C_{b}(X)^{\prime}$ is said to be $\sigma$-additive if $\Phi\left(u_{n}\right) \rightarrow 0$ for each sequence $\left(u_{n}\right)$ in $C_{b}(X)$ such that $u_{n}(x) \downarrow 0$ for all $x \in X$. We will denote by $L_{\sigma}\left(C_{b}(X)\right)$ the set of all $\sigma$-additive functionals on $C_{b}(X)$.

In the topological measure theory the so-called strict topologies on $C_{b}(X)$ are of importance (see $[17,21]$ for more details). In this paper we will consider the strict topology $\beta_{\sigma}$ on $C_{b}(X)$. Note that Sentilles [17] used for $\beta_{\sigma}$ the name superstrict topology and denoted it by $\beta_{1}$.

Now we recall the concept of the strict topology $\beta_{\sigma}$ on $C_{b}(X)$. Let $\beta X$ stand for the Stone-Čech compactification of $X$. For $v \in C_{b}(X), \bar{v}$ denotes its unique continuous extension to $\beta X$. For a compact subset $Q$ of $\beta X \backslash X$ let $C_{Q}(X)=\left\{v \in C_{b}(X)\right.$ : $\left.\left.\bar{v}\right|_{Q} \equiv 0\right\}$. Let $\beta_{Q}$ be the locally convex topology on $C_{b}(X)$ defined by the family of seminorms $\left\{p_{v}: v \in C_{Q}(X)\right\}$, where $p_{v}(u)=\sup _{x \in X}|u(x) v(x)|$ for $u \in C_{b}(X)$. We define the strict topology $\beta_{\sigma}$ on $C_{b}(X)$ to be the inductive limit topology $\operatorname{Lin} \beta_{Z}$ of the topologies $\beta_{Z}$ taken over the family $\mathcal{Z}$ of all zero sets such that $Z \subset \beta X \backslash X$ (see [17, pp. 314-315]).

It is known that $\beta_{\sigma}$ is a locally convex-solid topology and is a $\sigma$-Dini topology, that is, $u_{n} \rightarrow 0$ for $\beta_{\sigma}$ whenever $u_{n}(x) \downarrow 0$ for all $x \in X$ (see [17, Theorem 6.2], [21, Theorem 11.16]). Then

$$
\left(C_{b}(X), \beta_{\sigma}\right)^{\prime}=\left\{\Phi_{\mu}: \mu \in M_{\sigma}(X)\right\}=L_{\sigma}\left(C_{b}(X)\right),
$$

where $M_{\sigma}(X)$ stands for the space of $\sigma$-additive Baire measures (see [21, §4]). Moreover, $\left(C_{b}(X), \beta_{\sigma}\right)$ is a strong Mackey space, i.e., each countably $\sigma\left(M_{\sigma}(X)\right.$, $C_{b}(X)$ )-compact subset of $M_{\sigma}(X)$ is $\beta_{\sigma}$-equicontinuous (see [21, Theorem 11.5]). It follows that $\beta_{\sigma}=\tau\left(C_{b}(X), M_{\sigma}(X)\right)$. It is known that $\beta_{\sigma}$ is the finest locally convex topology on $C_{b}(X)$ which agrees with itself on $\|\cdot\|_{\infty}$-bounded (equivalently, $\beta_{\sigma}$-bounded) sets (see [17, Theorem 4.1], [21, Theorem 11.2]). This means that $\left(C_{b}(X), \beta_{\sigma}\right)$ is a generalized DF-space (see $\left.[15, \S 1]\right)$.

In this paper we study linear operators from $C_{b}(X)$ provided with $\beta_{\sigma}$ to $E$. In particular, we derive a Yosida-Hewitt type decomposition for weakly compact operators from $C_{b}(X)$ to $E$.

\section{Linear operators on $C_{b}(X)$ with the strict topology $\beta_{\sigma}$}

It is section we study linear operators from $C_{b}(X)$ (provided with the strict topology $\left.\beta_{\sigma}\right)$ to a Banach space $E$. For a bounded linear operator $T: C_{b}(X) \longrightarrow E$ 
let $T^{\prime}: E^{\prime} \longrightarrow C_{b}(X)^{\prime}$ denote its conjugate, i.e., $\left\langle u, T^{\prime}\left(e^{\prime}\right)\right\rangle=\left\langle T(u), e^{\prime}\right\rangle$ for $u \in C_{b}(X)$ and $e^{\prime} \in E^{\prime}$.

Definition 2.1 A bounded linear operator $T: C_{b}(X) \rightarrow E$ is said to be $\sigma$-additive if $\left\|T\left(u_{n}\right)\right\|_{E} \rightarrow 0$ for each sequence $\left(u_{n}\right)$ in $C_{b}(X)$ such that $u_{n}(x) \downarrow 0$ for all $x \in X$.

Now we prove a characterization of $\sigma$-additive operators $T: C_{b}(X) \longrightarrow E$.

Proposition 2.1 For a bounded linear operator $T: C_{b}(X) \longrightarrow E$ the following statements are equivalent:

(i) $T^{\prime}\left(E^{\prime}\right) \subset L_{\sigma}\left(C_{b}(X)\right)$ i.e., $e^{\prime} \circ T \in L_{\sigma}\left(C_{b}(X)\right)$ for each $e^{\prime} \in E^{\prime}$.

(ii) $T$ is $\left(\sigma\left(C_{b}(X), M_{\sigma}(X)\right), \sigma\left(E, E^{\prime}\right)\right)$-continuous.

(iii) $T$ is $\left(\beta_{\sigma},\|\cdot\|_{E}\right)$-continuous.

(iv) $T$ is sequentially $\left(\beta_{\sigma},\|\cdot\|_{E}\right)$-continuous.

(v) $T$ is $\sigma$-additive.

Proof (i) $\Longleftrightarrow$ (ii) See [1, Theorem 9.26].

(ii) $\Longleftrightarrow$ (iii) It is known that $T$ is $\left(\sigma\left(C_{b}(X), M_{\sigma}(X)\right), \sigma\left(E, E^{\prime}\right)\right)$-continuous if and only if $T$ is $\left(\tau\left(C_{b}(X), M_{\sigma}(X)\right),\|\cdot\|_{E}\right)$-continuous (see [1, Ex. 11, p. 149]). Since $\beta_{\sigma}=\tau\left(C_{b}(X), M_{\sigma}(X)\right)$, the proof is complete.

(iii) $\Longrightarrow$ (iv) It is obvious.

(iv) $\Longrightarrow$ (v) Assume that $T$ is sequentially $\left(\beta_{\sigma},\|\cdot\|_{E}\right)$-continuous, and let $\left(u_{n}\right)$ be a sequence in $C_{b}(X)$ such that $u_{n}(x) \downarrow 0$ for all $x \in X$. Since $\beta_{\sigma}$ is a $\sigma$-Dini topology, we get $u_{n} \rightarrow 0$ for $\beta_{\sigma}$. Hence $\left\|T\left(u_{n}\right)\right\|_{E} \longrightarrow 0$.

(v) $\Longrightarrow$ (i) It is obvious.

Let $\xi$ be a linear topology on $C_{b}(X)$. Recall that a linear operator $T: C_{b}(X) \longrightarrow E$ is said to be $\left(\xi,\|\cdot\|_{E}\right)$-weakly compact if there exists a neighbourhood $V$ of zero for $\xi$ such that $T(V)$ is a relatively $\sigma\left(E, E^{\prime}\right)$-compact subset of $E$. From now on we will briefly say that $T$ is weakly compact whenever $T$ is $\left(\|\cdot\|_{\infty},\|\cdot\|_{E}\right)$-weakly compact.

Proposition 2.2 For a linear operator $T: C_{b}(X) \longrightarrow E$ the following statements are equivalent:

(i) $T$ is weakly compact and $\left(\beta_{\sigma},\|\cdot\|_{E}\right)$-continuous.

(ii) $T$ is $\left(\beta_{\sigma},\|\cdot\|_{E}\right)$-weakly compact.

Proof (i) $\Longrightarrow$ (ii) Assume that $T$ is weakly compact and $\left(\beta_{\sigma},\|\cdot\|_{E}\right)$-continuous. Since $\beta_{\sigma}$-bounded subsets of $C_{b}(X)$ are $\|\cdot\|_{\infty}$-bounded, $T$ transforms $\beta_{\sigma}$-bounded sets into relatively $\sigma\left(E, E^{\prime}\right)$-compact sets in $E$. But $\left(C_{b}(X), \beta_{\sigma}\right)$ is a generalized DF-space, so in view of [15, Theorem 3.1] $T$ is $\left(\beta_{\sigma},\|\cdot\|_{E}\right)$-weakly compact, as desired.

(ii) $\Longrightarrow$ (i) Assume that $T$ is $\left(\beta_{\sigma},\|\cdot\|_{E}\right)$-weakly compact, i.e., there exists a neighbourhood $V$ of zero for $\beta_{\sigma}$ such that $T(V)$ is a relatively $\sigma\left(E, E^{\prime}\right)$-compact set in $E$. Hence $T(V) \subset B_{E}(r)\left(=\left\{e \in E:\|e\|_{E} \leq r\right\}\right)$ for some $r>0$. Then for $\varepsilon>0$ we get $T\left(\frac{\varepsilon}{r} V\right) \subset B_{E}(\varepsilon)$, and it follows that $T$ is $\left(\beta_{\sigma},\|\cdot\|_{E}\right)$-continuous. Clearly $T$ is weakly compact because $\beta_{\sigma}$ is weaker than the $\|\cdot\|_{\infty}$-topology.

Corollary 2.3 Assume that a linear operator $T: C_{b}(X) \longrightarrow E$ is $\left(\beta_{\sigma},\|\cdot\|_{E}\right)$-continuous. Then the following statements are equivalent: 
(i) $T$ is weakly compact.

(ii) $T$ is $\left(\beta_{\sigma},\|\cdot\|_{E}\right)$-weakly compact.

Remark It is well known (see [17]) that the strict topologies $\beta_{t}$ and $\beta_{\tau}$ on $C_{b}(X)$ both coincide with the Buck's original topology $\beta$ (see [9]) in the locally compact case. Sentilles [16] showed that if $T$ is a $\left(\beta,\|\cdot\|_{E}\right)$-continuous linear operator from $C_{b}(X)$ ( $X$ is locally compact) to $E$, then $T$ is weakly compact if and only if $T$ is $\left(\beta,\|\cdot\|_{E}\right)$-weakly compact.

Let $B(\mathcal{B})$ (resp. $B(\mathcal{B} a)$ ) denote the Banach space of all totally $\mathcal{B}$-measurable (respectively, totally $\mathcal{B} a$-measurable) functions $u: X \rightarrow \mathbb{R}$, provided with the uniform norm $\|\cdot\|_{\infty}($ see $[11, \S 6]) .\left(B(\mathcal{B} a),\|\cdot\|_{\infty}\right)$ is a $\sigma$-Dedekind complete AM-space (see [3, Theorem 13.2]). Then $C_{b}(X) \subset B(\mathcal{B})$ (see [4, Lemma 1.2]) and one can inject isometrically $B(\mathcal{B})$ into $C_{b}(X)^{\prime \prime}$ by the mapping $\pi: B(\mathcal{B}) \longrightarrow C_{b}(X)^{\prime \prime}$, where for each $u \in B(\mathcal{B})$

$$
\pi(u)\left(\Phi_{\mu}\right)=\int_{X} u(x) \mathrm{d} \mu \text { for all } \mu \in M(X) .
$$

Let $i: E \rightarrow E^{\prime \prime}$ stand for the canonical isometry, i.e., $i(e)\left(e^{\prime}\right)=e^{\prime}(e)$ for all $e \in E$ and $e^{\prime} \in E^{\prime}$. Moreover, let $j: i(E) \longrightarrow E$ denote the left inverse of $i$, i.e., $j \circ i=i d_{E}$.

Now assume that $T: C_{b}(X) \rightarrow E$ is a weakly compact operator. Let $T^{\prime \prime}$ : $C_{b}(X)^{\prime \prime} \longrightarrow E^{\prime \prime}$ denote the biconjugate of $T$. Then $T^{\prime \prime}\left(C_{b}(X)^{\prime \prime}\right) \subset i(E)$ (see $[1$, Theorem 17.2]) and we can define a representing measure $m: \mathcal{B} \rightarrow E$ by

$$
m(A):=j\left(T^{\prime \prime}\left(\pi\left(\mathbb{1}_{A}\right)\right)\right) \text { for all } A \in \mathcal{B}
$$

(here $\mathbb{1}_{A}$ stands for the characteristic function of a set $A$ ). Using the Alexandroff representation theorem (see [21, Theorem 5.1]) we obtain that for each $e^{\prime} \in E^{\prime}, e^{\prime} \circ m \in$ $M(X)$ and

$$
e^{\prime}(T(u))=\int_{X} u(x) d\left(e^{\prime} \circ m\right) \text { for all } u \in C_{b}(X) .
$$

Let

$$
\widetilde{T}:=j \circ T^{\prime \prime} \circ \pi: B(\mathcal{B}) \longrightarrow E .
$$

Then $\widetilde{T}$ is a bounded linear operator and

$$
\widetilde{T}(u)=\int_{X} u(x) \mathrm{d} m \text { for all } u \in B(\mathcal{B}),
$$


and $\|\widetilde{T}\|=\|m\|(X)$ (= the semivariation of $m$ ) (see [10, Theorem 1.1.13]). Since $T^{\prime \prime}: C_{b}(X)^{\prime \prime} \longrightarrow E^{\prime \prime}$ is a weakly compact operator (see [1, Theorem 17.2]) and the mapping $j: i(E) \rightarrow E$ is $\left(\sigma\left(i(E), E^{\prime}\right), \sigma\left(E, E^{\prime}\right)\right)$-continuous, we see that $\widetilde{T}$ is also weakly compact. It follows that $m$ is strongly bounded (see [10, Theorem 6.1.1]). One can easily verify that

$$
T(u)=\widetilde{T}(u)=\int_{X} u(x) \mathrm{d} m \text { for all } u \in C_{b}(X) .
$$

It is well known that the $\sigma$-order continuous dual $B(\mathcal{B} a)_{c}^{\sim}$ of $B(\mathcal{B} a)$ can be identified with the Banach lattice $c a(\mathcal{B} a)$ of countably additive signed measures on $\mathcal{B} a$ throughout the lattice isomorphism $c a(\mathcal{B} a) \ni v \mapsto \Phi_{v} \in B(\mathcal{B} a)_{c}^{\sim}$, where $\Phi_{v}(u)=$ $\int_{X} u(x) d v$ for all $u \in B(\mathcal{B} a)$ (see [3, Theorem 13.5]). Then the Mackey topology $\tau(B(\mathcal{B} a), c a(\mathcal{B} a)$ ) is a locally solid $\sigma$-Lebesgue topology on $B(\mathcal{B} a$ ) (see [? Ex. 18 , p. 178]AB2) and $(B(\mathcal{B} a), \tau(B(\mathcal{B} a), c a(\mathcal{B} a)))$ is a generalized DF-space (see [13, $\S 4])$.

It follows that

$$
\left.\tau(B(\mathcal{B} a), c a(\mathcal{B} a))\right|_{C_{b}(X)} \subset \beta_{\sigma}=\tau\left(C_{b}(X), M_{\sigma}(X)\right) .
$$

Indeed, let $\left(u_{n}\right)$ be a sequence in $C_{b}(X)$ and $u_{n}(x) \downarrow 0$ for all $x \in X$. Then $u_{n} \downarrow 0$ in $B(\mathcal{B} a)$ (in the vector lattice sense); hence $u_{n} \rightarrow 0$ for $\tau(B(\mathcal{B} a), c a(\mathcal{B} a)$ ). This means that $\left.\tau(B(\mathcal{B} a), c a(\mathcal{B} a))\right|_{C_{b}(X)}$ is a $\sigma$-Dini topology on $C_{b}(X)$. Since $\beta_{\sigma}$ is the finest $\sigma$-Dini topology on $C_{b}(X)$ (see [[21, Theorem 11.16]), we conclude that the inclusion (2.2) holds.

Now we are ready to state the following result.

Proposition 2.4 Let $T: C_{b}(X) \rightarrow$ E be a $\left(\beta_{\sigma},\|\cdot\|_{E}\right)$-weakly compact linear operator, and let $m: \mathcal{B} \rightarrow E$ be its representing measure. Then $m$ has a unique countably additive extension $\bar{m}: \mathcal{B} a \rightarrow E$ and the corresponding integration operator $T_{\bar{m}}$ : $B(\mathcal{B} a) \rightarrow E$ is $\left(\tau(B(\mathcal{B} a), c a(\mathcal{B} a)),\|\cdot\|_{E}\right)$-weakly compact and $T_{\bar{m}}(u)=\widetilde{T}(u)=$ $T(u)$ for $u \in C_{b}(X)$.

Proof In view of Proposition $2.1 e^{\prime} \circ T \in L_{\sigma}\left(C_{b}(X)\right)$ for each $e^{\prime} \in E^{\prime}$. Hence by (2.1) and (1.1) we obtain that $e^{\prime} \circ m \in M_{\sigma}(X)$ for each $e^{\prime} \in E^{\prime}$. This means that $e^{\prime} \circ m: \mathcal{B} \rightarrow \mathbb{R}$ is countably additive for each $e^{\prime} \in E^{\prime}$. Since $m: \mathcal{B} \rightarrow E$ is strongly bounded, by the Carathéodory-Hahn-Kluvanek extension theorem (see [10, Theorem 1.5.2]) $m$ has a unique countably additive extension $\bar{m}: \mathcal{B} a \rightarrow E$. Next, by [13, Corollary 12] the corresponding integration operator $T_{\bar{m}}: B(\mathcal{B a}) \rightarrow E$ is $\left(\tau(B(\mathcal{B} a), c a(\mathcal{B} a)),\|\cdot\|_{E}\right)$-weakly compact.

Now let $u \in C_{b}(X) \subset B(\mathcal{B}) \subset B(\mathcal{B} a)$. Then there exists a sequence $\left(s_{n}\right)$ of $\mathcal{B}$-simple functions such that $\left\|s_{n}-u\right\|_{\infty} \rightarrow 0$. Hence we get $T_{\bar{m}}(u)=\lim _{n} T_{\bar{m}}\left(s_{n}\right)=$ $\lim _{n} T_{m}\left(s_{n}\right)=\widetilde{T}(u)=T(u)$. Thus the proof is complete. 


\section{Yosida-Hewitt type decomposition for weakly compact operators on $C_{b}(X)$}

There are many versions and generalizations of the classical Yosida-Hewitt decomposition theorem [22] in diverse setting, e.g. for vector-valued and group valued measures (see $[7,19,14,18,12])$, operators between vector-lattices and order-weakly compact operators from vector lattices to Banach spaces (see [5,6]). Brooks and Wright [8] obtained a Yosida-Hewitt type decomposition for weakly compact operators on a von Neumann algebra $\mathcal{M}$. In this section we derive a Yosida-Hewitt type decomposition for weakly compact operators from $C_{b}(X)$ to a Banach space $E$.

Since $M(X)$ is a Dedekind complete vector lattice and $M_{\sigma}(X)$ is a band of $M(X)$ (see [21, Theorem 7.2]), we see that $M_{\sigma}(X)$ is a projective band of $M(X)$ (see [[1, Theorem 3.8]). Thus we obtain the following Yosida-Hewitt decomposition

$$
M(X)=M_{\sigma}(X) \oplus M_{p f a}(X),
$$

where $M_{p f a}(X)\left(=M_{\sigma}(X)^{d}\right.$ - the disjoint complement of $M_{\sigma}(X)$ in $\left.M(X)\right)$ stands for the space of purely finitely additive members of $M(X)$. Hence

$$
C_{b}(X)^{\prime}=L_{\sigma}\left(C_{b}(X)\right) \oplus L_{p f a}\left(C_{b}(X)\right),
$$

where $L_{p f a}\left(C_{b}(X)\right)\left(=L_{\sigma}\left(C_{b}(X)\right)^{d}\right.$-the disjoint complement of $L_{\sigma}\left(C_{b}(X)\right)$ in $\left.C_{b}(X)^{\prime}\right)$ stands for the space of purely finitely additive functionals in $C_{b}(X)^{\prime}$. Since $C_{b}(X)$ is a AM-space, $M(X)$ is a AL-space. This means that $\|\mu\|=\left\|\mu_{c}\right\|+\left\|\mu_{p}\right\|$ and $\left\|\Phi_{\mu}\right\|=\left\|\Phi_{\mu_{c}}\right\|+\left\|\Phi_{\mu_{p}}\right\|$ when $\mu=\mu_{c}+\mu_{p}$ with $\mu_{c} \in M_{\sigma}(X)$ and $\mu_{p} \in M_{p f a}(X)$.

Remark Note that if $X$ is pseudocompact, then $\beta_{\sigma}$ coincides with the $\|\cdot\|_{\infty}$-topology and hence $M_{\sigma}(X)=M(X)$. Then $L_{\sigma}\left(C_{b}(X)\right)=C_{b}(X)^{\prime}$ and $L_{p f a}\left(C_{b}(X)\right)=\{0\}$.

Definition 3.1 A bounded linear operator $T: C_{b}(X) \longrightarrow E$ is said to be purely finitely additive if $e^{\prime} \circ T \in L_{p f a}\left(C_{b}(X)\right)$ for each $e^{\prime} \in E^{\prime}$.

Now we are in position to prove a Yosida-Hewitt type decomposition for weakly compact operators from $C_{b}(X)$ to $E$.

Theorem 3.1 Let $T: C_{b}(X) \longrightarrow$ E be a weakly compact operator and let $m: \mathcal{B} \longrightarrow$ $E$ be its representing measure. Then

(i) $m$ can be uniquely decomposed as $m=m_{c}+m_{p}$, where $m_{c}: \mathcal{B} \longrightarrow E$ and $m_{p}$ : $\mathcal{B} \longrightarrow E$ are strongly bounded measures, and $e^{\prime} \circ m_{c} \in M_{\sigma}(X)$ (hence $m_{c}$ has a unique countably additive extension $\left.\overline{m_{c}}: \mathcal{B} a \longrightarrow E\right)$ and $e^{\prime} \circ m_{p} \in M_{p f a}(X)$ for each $e^{\prime} \in E^{\prime}$.

(ii) $T$ can be uniquely decomposed as $T=T_{1}+T_{2}$, where $T_{1}$ and $T_{2}$ are weakly compact operators, $T_{1}$ is $\sigma$-additive (hence $T_{1}$ is $\left(\beta_{\sigma},\|\cdot\|_{E}\right)$-weakly compact) and $T_{2}$ is purely finitely additive, and

$$
T_{1}(u)=\int_{X} u(x) \mathrm{d} m_{c} \text { and } T_{2}(u)=\int_{X} u(x) \mathrm{d} m_{p} \text { forall } u \in C_{b}(X) .
$$


In consequence,

$$
T(u)=\int_{X} u(x) \mathrm{d} m=\int_{X} u(x) \mathrm{d} m_{c}+\int_{X} u(x) \mathrm{d} m_{p} \text { for all } u \in C_{b}(X) .
$$

Proof We have $C_{b}(X)^{\prime}=L_{\sigma}\left(C_{b}(X)\right) \oplus L_{p f a}\left(C_{b}(X)\right)$ and $\|\Phi\|=\left\|\Phi_{1}\right\|+\left\|\Phi_{2}\right\|$, where $\Phi_{1} \in L_{\sigma}\left(C_{b}(X)\right)$ and $\Phi_{2} \in L_{p f a}\left(C_{b}(X)\right)$. Thus we have natural projections

$$
P_{k}: C_{b}(X)^{\prime} \longrightarrow C_{b}(X)^{\prime}
$$

where $P_{k}(\Phi)=\Phi_{k}$ and $\left\|P_{k}\right\| \leq 1(k=1,2)$. Now we can consider the conjugate operators

$$
P_{k}^{\prime}: C_{b}(X)^{\prime \prime} \longrightarrow C_{b}(X)^{\prime \prime}
$$

defined by $P_{k}^{\prime}(V)(\Phi)=V\left(P_{k}(\Phi)\right)$ for $V \in C_{b}(X)^{\prime \prime}$ and $\Phi \in C_{b}(X)^{\prime}$. One can observe that $\left(P_{k}^{\prime} \circ \pi\right)(u)=\pi(u) \circ P_{k}$ for all $u \in B(\mathcal{B})$. Define linear operators $(k=1,2)$

$$
\widetilde{T}_{k}:=j \circ T^{\prime \prime} \circ P_{k}^{\prime} \circ \pi: B(\mathcal{B}) \longrightarrow E .
$$

Since $T^{\prime \prime}: C_{b}(X)^{\prime \prime} \longrightarrow E^{\prime \prime}$ is a weakly compact operator with $T^{\prime \prime}\left(C_{b}(X)^{\prime \prime}\right) \subseteq$ $i(E)$, and the mapping $j$ is $\left.\left(\sigma\left(i(E), E^{\prime}\right)\right), \sigma\left(E^{\prime}, E\right)\right)$-continuous, we obtain that $\widetilde{T}_{k}$ are weakly compact.

Let $m_{c}(A):=\widetilde{T}_{1}\left(\mathbb{1}_{A}\right)$ and $m_{p}(A):=\widetilde{T}_{2}\left(\mathbb{1}_{A}\right)$ for $A \in \mathcal{B}$. Hence $m_{c}: \mathcal{B} \longrightarrow E$ and $m_{p}: \mathcal{B} \longrightarrow E$ are strongly bounded measures and $\left\|m_{c}\right\|(X)=\left\|\widetilde{T}_{1}\right\|$ and $\left\|m_{p}\right\|(X)=$ $\left\|\widetilde{T}_{2}\right\|$. Moreover, for all $u \in B(\mathcal{B})$ we have

$$
\widetilde{T}_{1}(u)=\int_{X} u(x) \mathrm{d} m_{c}, \quad \widetilde{T}_{2}(u)=\int_{X} u(x) \mathrm{d} m_{p} .
$$

Note that $\widetilde{T}(u)=\widetilde{T}_{1}(u)+\widetilde{T}_{2}(u)$ for all $u \in B(\mathcal{B})$. Hence

$$
m(A)=m_{c}(A)+m_{p}(A) \text { for all } A \in \mathcal{B}
$$

For $k=1,2$ define $T_{k}=\left.\widetilde{T}_{k}\right|_{C_{b}(X)}: C_{b}(X) \longrightarrow E$. Then $T(u)=T_{1}(u)+T_{2}(u)$ for all $u \in C_{b}(X)$ and $T_{1}$ and $T_{2}$ are weakly compact.

For each $e^{\prime} \in E^{\prime}$ and all $u \in C_{b}(X)$ we have

$$
\begin{aligned}
\left(e^{\prime} \circ T_{k}\right)(u)=e^{\prime}\left(T_{k}(u)\right) & =\left(\left(T^{\prime \prime} \circ P_{k}^{\prime} \circ \pi\right)(u)\right)\left(e^{\prime}\right) \\
& =\left(T^{\prime \prime}\left(\pi(u) \circ P_{k}\right)\right)\left(e^{\prime}\right) \\
& =\left(\pi(u) \circ P_{k}\right)\left(T^{\prime}\left(e^{\prime}\right)\right) \\
& =\pi(u)\left(P_{k}\left(e^{\prime} \circ T\right)\right) \\
& =P_{k}\left(e^{\prime} \circ T\right)(u) .
\end{aligned}
$$


Since $e^{\prime} \circ T \in C_{b}(X)^{\prime}=L_{\sigma}\left(C_{b}(X)\right) \oplus L_{p f a}\left(C_{b}(X)\right)$, we get $e^{\prime} \circ T_{1} \in L_{\sigma}\left(C_{b}(X)\right)$ and $e^{\prime} \circ T_{2} \in L_{p f a}\left(C_{b}(X)\right)$. In view of Proposition 2.1, $T_{1}$ is $\sigma$-additive and $T_{2}$ is purely finitely additive. Moreover, by Proposition $2.2, T_{1}$ is $\left(\beta_{\sigma},\|\cdot\|_{E}\right)$-weakly compact. The uniquenes of the decomposition $T=T_{1}+T_{2}$ follows from the uniqueness of the decomposition $e^{\prime} \circ T=e^{\prime} \circ T_{1}+e^{\prime} \circ T_{2}$ for each $e^{\prime} \in E^{\prime}$. Moreover, in view of (3.1) for $u \in C_{b}(X)$ we have

$$
\left(e^{\prime} \circ T_{1}\right)(u)=\int_{X} u(x) \mathrm{d}\left(e^{\prime} \circ m_{c}\right) \text { and }\left(e^{\prime} \circ T_{2}\right)(u)=\int_{X} u(x) \mathrm{d}\left(e^{\prime} \circ m_{p}\right) .
$$

Hence $e^{\prime} \circ m_{c} \in M_{\sigma}(X)$ and $e^{\prime} \circ m_{p} \in M_{p f a}(X)$. By Proposition $2.4 m_{c}$ has a unique countably additive extension $\overline{m_{c}}: \mathcal{B} a \longrightarrow E$.

Acknowledgments The author is grateful to the referee for the valuable remarks.

Open Access This article is distributed under the terms of the Creative Commons Attribution Noncommercial License which permits any noncommercial use, distribution, and reproduction in any medium, provided the original author(s) and source are credited.

\section{References}

1. Aliprantis, C.D., Burkinshaw, O.: Positive Operators. Academic Press, New York (1985)

2. Aliprantis, C.D., Burkinshaw, O.: Locally Solid Riesz Spaces with Applications to Economics, 2nd edn, Math. Surveys and Monographs, no. 105, 2003.

3. Aliprantis, C.D., Border, K.C.: Infinite Dimensional Analysis, 2nd edn. Springer, Berlin (1999)

4. Aguayo, J., Sanchez, J.: Weakly compact operators and the strict topologies. Bull. Austral. Math. Soc. 39, 353-359 (1989)

5. Basile, A., Bukhvalov, A.V., Yakubson, M.Ya.: The generalized Yosida-Hewitt theorem. Math. Proc. Camb. Phil. Soc. 116, 527-533 (1994)

6. Basile, A., Bukhvalov, A.: On a Unifying Approach to Decomposition Theorems of Yosida-Hewitt Type. Ann. Matematica Pura Appl. 173(4), 107-125 (1997)

7. Brooks, J.K.: Decomposition theorems for vector measures. Proc. Am. Math. Soc. 21, 27-29 (1969)

8. Brooks, J.K., Wright, J.D.M.: Representing Yosida-Hewitt decompositions for classical and non-commutatitve vector measures. Expo. Math. 19, 373-383 (2001)

9. Buck, R.C.: Bounded continuous functions on a locally compact space. J. Mich. Math. 6, 95-104 (1958)

10. Diestel, J., Uhl, J.J.: Vector Measures. Am. Math. Soc. Math. Surveys 15, Providence, RI (1977)

11. Dinculeanu, N.: Vector Measures. Pergamon Press, New York (1967)

12. Drewnowski, L.: Decompositions of set functions. Studia Math 58, 23-48 (1973)

13. Graves, W.H., Ruess, W.: Compactness in spaces of vector-valued measures and a natural Mackey topology for spaces of bounded measurable functions. Contemp. Math. 2, 189-203 (1980)

14. Huff, R.E.: The Yosida-Hewitt decomposition as an ergodic theorem, In: Tucker, D.H., Maynard, H.B. (eds.) Vector and Operator-valued Measures and Applications, pp. 133-139. Academic Press, New York (1973)

15. Ruess, W.: [Weakly] compact operators and DF-spaces. Pacific J. Math. 98(2), 419-441 (1982)

16. Sentilles, F.D.: Compact and weakly compact operators on $C(S)_{\beta}$, Illin. J. Math. 13, 769-776 (1969)

17. Sentilles, F.D.: Bounded continuous functions on a completely regular spaces. Trans. Am. Math. Soc. 168, 311-336 (1972)

18. Traynor, T.: A general Hewitt-Yosida decomposition. Can. J. Math. 24(6), 1164-1169 (1972)

19. Uhl, J.J.: Extensions and decompositions of vector measures. J. Lond. Math. Soc. 2(2), 672-676 (1971) 
20. Varadarajan, V.S.: Measures on topological spaces, Mat. Sbornik (N.S.) 55 (97), 35-100 (1961). Am. Math. Soc. Transl. (2) 48, 161-228 (1965)

21. Wheeler, R.: A survey of Baire measures and strict topologies. Expo. Math. 1, 97-190 (1983)

22. Yosida, K., Hewitt, E.: Finitely additive measures. Trans. Am. Math. Soc. 72, 46-66 (1952) 